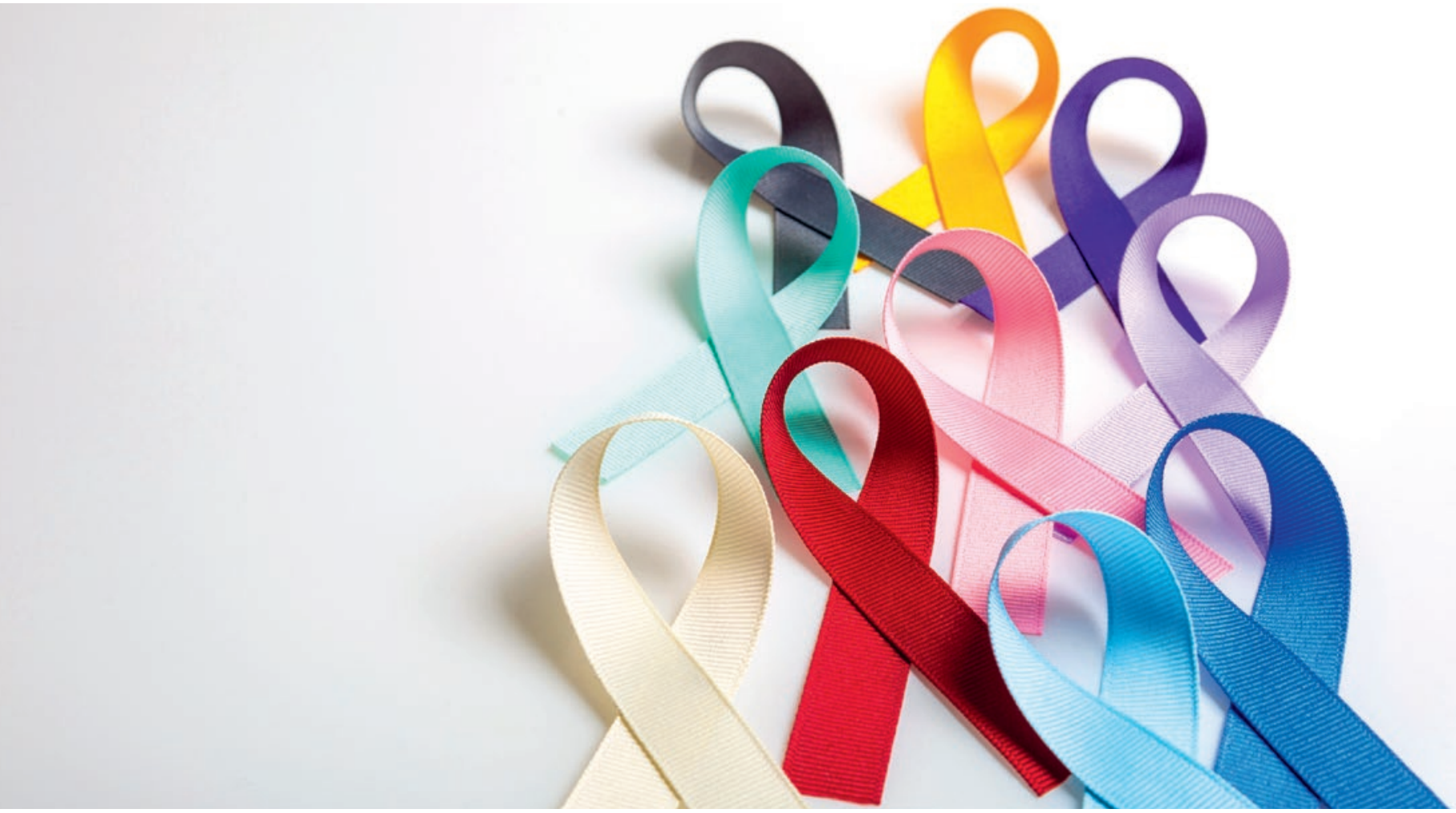

\title{
Die Krebsliga Ostschweiz und die regionale Onkologie
}

Théo Buff ${ }^{a}$, Urs Weishaupt ${ }^{\mathrm{b}}$, Regula Schneider ${ }^{\mathrm{c}}$, Rudolf Morant ${ }^{\mathrm{d}}$

${ }^{a}$ lic. phil., Historiker und freischaffender Autor, St. Gallen; ${ }^{b}$ Vorstandsmitglied emeritus Krebsliga Ostschweiz (KLOCH), St. Gallen; ${ }^{c}$ Geschäftsführerin KLOCH; ${ }^{d}$ Dr. med., Innere Medizin, FMH, spez. Hämatologie/Onkologie, Präsident KLOCH

Die Geschichte der Krebsliga Ostschweiz zeigt, welch grosse Wirkung der Einsatz Einzelner für die Interessen von Krebsbetroffenen entfalten konnte: So betrieb die Krebsliga eines der ersten zytologischen Labors, gründete das erste Krebsregister der Schweiz, gab den Anstoss zu einer onkologischen Abteilung am Kantonsspital St. Gallen und förderte dann deren Weiterentwicklung massgeblich.

\section{Jahre Krebsliga Ostschweiz}

Anlässlich des 65. Geburtstags der Krebsliga Ostschweiz hat der Historiker Théo Buff die vorhandenen Unterlagen zusammengetragen, gesichtet und im Kontext soweit möglich verifiziert.

Am Anfang der spannenden Geschichte, die zutage gefördert wurde, stehen Individuen und Visionäre mit einer kleinen Wohltätigkeitsorganisation. Diese hat sich über die Jahrzehnte zur heutigen stabilen und professionell geführten Institution entwickelt mit vielfältigen
Aufgaben in der Betreuung von Krebsbetroffenen und deren Angehörigen in allen Krankheitsphasen, in der Krebsregistrierung und Vorsorge von Krebserkrankungen. Diese insgesamt erfreuliche Entwicklung verlief nicht gradlinig, sondern war zeitweilig durch harte Richtungskämpfe, Zank und Enttäuschungen geprägt. In diesem Artikel beschränken wir uns auf einen Aspekt, der sich bei der Sichtung der Unterlagen als wichtige, für manche auch überraschende Erkenntnis herauskristallisiert hat: Die lokale Krebsliga war massgeblich an der Entstehung und Entwicklung der Onko- 
logie in Spital, Forschung, Kongresswesen und Praxis in der Ostschweiz beteiligt. Dies dank starken Persönlichkeiten, die sich bei Ärztinnen und Ärzten, in der Politik und in der Öffentlichkeit wirkungsvoll für ihre Anliegen eingesetzt hatten. Der anfängliche Fokus lag auf Forschung und Therapie, wodurch die direkte Arbeit mit Patientinnen und Patienten noch etwas im Hintergrund stand. Diese Tätigkeiten und die Vorsorge wurden dafür in der weiteren Entwicklung umso bedeutender.

\section{Gründung des zytologischen Laboratoriums}

Die «Krebsliga für St.Gallen und beide Appenzell» (im weiteren Krebsliga genannt) wurde vom Gynäkologen Dr. Franz Iklé zur Wahrung der Interessen von Krebskranken, zur Förderung der Vorsorge und zur besseren Behandlung und Betreuung von Betroffenen 1956 gegründet. Er blieb Präsident der Liga bis 1964.

In jenen Jahren sind in verschiedenen Kantonen der Schweiz ähnliche Ligen gegründet worden. Die Vorläuferin der heutigen Dachorganisation, die «Krebsliga Schweiz», war hingegen bereits 1910 entstanden. Dr. Iklé hatte schon 1952 die "Abstrichzytologie» im Labor für gynäkologische Zytologie an der Frauenklinik eingeführt. Daraus entstand in Zusammenarbeit mit dem Kanton St.Gallen das zytologische Laboratorium, welches er 1958 in die Krebsliga überführen konnte. Durch die Verbreitung regelmässiger Vorsorgeuntersuchungen und einer qualitativ hochstehenden zytologischen Beurteilung haben Dr. Iklé und damit die Krebsliga massgeblich zur Früherkennung des Zervixkarzinoms beigetragen. Lange Zeit bildeten die Einnahmen dieses Labors die finanzielle Grundlage der Krebsliga. 2002 wurde dieses Labor in den Räumen der Krebsliga aufgehoben und in die $\mathrm{Pa}$ thologie am Kantonsspital St. Gallen überführt.

\section{Erstes Krebsregister der Schweiz}

Ein zweites Pionierprojekt der jungen Krebsliga war das Krebsregister. Es wurde 1960 von den Ärzten Dr. Iklé, Forster und Zollinger als Zettelkastenregister begonnen. Es war das erste seiner Art in der Schweiz. Auf der Pathologie wurden sämtliche durch Biopsien, Operationen oder Autopsien erfassten Krebsfälle dokumentiert. Das System galt bald als gesamtschweizerisch beispielhaft. Heute erfasst das Krebsregister Ostschweiz die Krebserkrankungen in den Kantonen St.Gallen, beider Appenzell, Thurgau und im Fürstentum Liechtenstein und ist fachlich verantwortlich für die Register in Graubünden und Glarus. Die Auswer- tung der Daten erlaubt wertvolle Einblicke in die Entwicklung der Krebshäufigkeit und Sterblichkeit und kann Ausgangspunkt für kantonale Massnahmen im Bereich der Prävention und Früherkennung sein. Es begleitet wissenschaftlich das ebenfalls von der Krebsliga Ostschweiz geführte Brustkrebs-Früherkennungsprogramm «donna».

\section{Kerngeschäft: Beratung und Unterstützung}

Ein Kerngeschäft der regionalen Krebsligen ist seit ihrer Gründung die kostenlose Beratung und Unterstützung von Krebsbetroffenen und Angehörigen. Dies wurde anfänglich von der als «Fürsorgerin» bezeichneten Ordensfrau Gertrud Hungerbühler übernommen. Aktuell betreibt die Krebsliga Ostschweiz Beratungsstellen in St.Gallen, Buchs und Glarus mit Sozialarbeiterinnen und -arbeitern sowie Pflegefachpersonen. Dazu gehört auch die spezialisierte Fachberatung Cancer Survivorship, welche sich der Linderung der Langzeitfolgen von Krebs verschrieben hat.

Das Krebsregister wurde 1960 als Zettelkastenregister begonnen und war das erste seiner Art in der Schweiz.

\section{Entstehung der Onkologie Ostschweiz}

1969 ergriff die Krebsliga die Initiative zur Gründung einer onkologischen Station am Kantonsspital für dieses neue, sich schnell entwickelnde Fachgebiet. Sie leistete dazu auch finanzielle Unterstützung. Daraufhin beschloss der Regierungsrat 1971 die Einrichtung einer onkologischen Station am Kantonsspital St.Gallen (KSSG) mit vorerst zehn Betten für akute Therapiephasen und zwei Sterilbetten. Dies gegen den Widerstand des Finanzdepartements. Die Station wurde 1972 eröffnet und vom aus Basel berufenen PD Dr. Hans-Jörg Senn geleitet, einem der Pioniere der Onkologie in der Schweiz. Die neu geschaffene Klinik C umfasste neben der Onkologie und Hämatologie mit den drei Schwerpunkten Bettenstation, Ambulatorium (ab 1973) und wissenschaftlichem Labor auch die Gastroenterologie. In engem Kontakt mit der Krebsliga gründete Prof. Dr Senn die Ostschweizerische Arbeitsgemeinschaft für klinische Onkologie (OSAKO). Diese organisierte regelmässige Treffen der Onkologinnen und Onkologen in der Ostschweiz, publizierte Behandlungs- und Nachsorgerichtlinien der häufigsten Tumoren und startete eine Studie zur adjuvanten zytostatischen Behandlung des frühen Mammakarzinoms. Diese frühe mutige 
Arbeit war anfänglich auch in Fachkreisen umstritten, wurde sogar öffentlich angegriffen, aber nachher international publiziert und wies den Weg für die Weiterentwicklung der heute erfolgreichen adjuvanten Therapie des Mammakarzinoms.

\section{Förderung St. Galler Kongresse}

Die interdisziplinäre Behandlung des frühen Mammakarzinoms wurde in regelmässigen wissenschaftlichen Kongressen auf internationalem Niveau diskutiert. Der erste dieser von Prof. Senn geleiteten und anfänglich von der Krebsliga finanziell geförderten Kongresse, von 70 Personen besucht, fand 1978 statt. Am zweiten Kongress 1984 waren bereits 225 Teilneh-

\section{Die Krebsliga hat die Früherkennung zu einem medizinischen und politischen Thema gemacht.}

mende anwesend, und 2009 war mit 4700 Teilnehmenden aus über 100 Ländern der Höhepunkt erreicht. Seit 2015 werden diese "St. Galler» Kongresse in Wien durchgeführt. Das jeweilige Ergebnis, der sogenannte «St.Galler Consensus» für die Therapie des frühen Mammakarzinoms, beinhaltet weltweit publizierte und beachtete Richtlinien für die Behandlung des frühen Mammakarzinoms. Er machte St.Gallen für mit Brustkrebs arbeitende Fachpersonen weltweit bekannt. Mit seiner Arbeitsaufnahme in St. Gallen wurde PD Dr. Senn einstimmig in den Vorstand der Krebsliga gewählt, was die weitere enge Zusammenarbeit prägte und auch die finanzielle Unterstützung der klinischen

\section{Das Wichtigste in Kürze}

- Die Krebsliga Ostschweiz (KLOCH) hat den Aufbau und die Weiterentwicklung onkologischer Angebote in St. Gallen ermöglicht. Dies durch die Einführung zytologischer Untersuchungen, die Gründung des ersten Krebsregisters der Schweiz, die Einrichtung einer onkologischen Abteilung und onkologischen Kongressen mit weltweiter Ausstrahlung

- $\quad$ Die $\mathrm{KLOCH}$ hat zudem durch den Aufbau eines palliativen Brückendiensts in Zusammenarbeit mit dem Palliativzentrum des Kantonsspitals massgeblich zur palliativen Pflege beigetragen.

Dr. med. Rudolf Morant

Krebsliga Ostschweiz

Flurhofstrasse 7

CH-9000 St. Gallen

rudolf.morant[at]krebsliga-

ostschweiz.ch

- Aktuell betreibt die $\mathrm{KLOCH}$ mit ihrem erfolgreichen Programm «donna» das schweizweit grösste Brustkrebs-Früherkennungsprogramm. Die Krebsliga ist sowohl als Impulsgeber wie auch als ausführende Institution ein wichtigerTeil der kantonalen Gesundheitspolitik.
Onkologie in allen Aspekten durch die Krebsliga gewährleistete.

Die Krebsliga übernimmt auch Aufgaben, die sonst nicht durch- oder weitergeführt werden. So übernahm sie 1992 die Stomaberatung vom Kantonsspital.

\section{Erstes Mammografie-Screening in der Deutschschweiz}

Die Krebsliga hatte das Thema Früherkennung aufs medizinische und politische Tableau gebracht und sich für die Einführung von Vorsorgeprogrammen eingesetzt. In der Folge beauftragte sie der Kanton St.Gallen 2009 mit einem Brustkrebs-Früherkennungsprogramm. Bereits seit elf Jahren betreibt die Krebsliga das überregional erfolgreiche und anerkannte Mammografie-Screening-Programm «donna». Frauen zwischen 50 und 69 Jahren erhalten von donna in zweijährlichen Abständen eine Einladung für eine franchisenbefreite Mammografie-Untersuchung. Dieses Früherkennungsprogramm ist das grösste seiner Art der Schweiz und weist eine durchschnittliche Teilnahmerate um die 50\% auf. Die Krebsliga betreibt es aktuell im Auftrag der Kantone St.Gallen, Graubünden, Bern und Solothurn.

Ein niederschwelliges Darmkrebs-Screening-Programm für den Kanton St.Gallen mit Direktversand eines immunologischen Blut-im-Stuhl-Tests (FIT-Test) ist derzeit in Planung.

Bildnachweis

Elmer Grubbs | Dreamstime.com (Symbolbild)

\section{L'essentiel en bref}

- La Ligue contre le cancer de Suisse orientale (KLOCH) a permis la création et le développement de services oncologiques à Saint-Gall. Cet objectif a été atteint grâce à l'introduction des examens cytologiques, la création du premier registre du cancer en Suisse, la mise en place d'un département d'oncologie et à des congrès d'oncologie de portée mondiale.

- $\mathrm{KLOCH}$ a fortement contribué aux soins palliatifs en mettant en place un service de soins palliatifs en collaboration avec le centre palliatif de l'hôpital cantonal.
Grâce au succès de son programme «donna», KLOCH mène actuellement le plus grand programme de dépistage du cancer du sein en Suisse. La Ligue contre le cancer est un élément important de la politique de santé cantonale, en tant qu'initiatrice et institution d'exécution. 